\title{
Manejo de artralgia de articulación temporomandibular mediante la utilización de dispositivos interoclusales tipo pívot; revisión de la literatura
}

\section{Management of arthralgia of temporomandibular joint through the use of interocclusal pivot splint; a review}

Cerda-Trujillo $\mathrm{C}^{*}$, Iturriaga $\mathrm{V}^{* *}$, Fuentes $\mathrm{R} * * *$, Bornhardt $\mathrm{T}^{* * * *}$

\section{RESUMEN}

Artralgia de la articulación temporomandibular (ATM) es un término utilizado en los criterios diagnósticos para los trastornos temporomandibulares (CD/TTM) definido como dolor de origen articular que se ve aumentado por el movimiento mandibular, ya sea en función o parafunción, y la replicación de este dolor mediante la palpación de la ATM. En relación al manejo de la artralgia, la evidencia describe diferentes tipos de terapias, las cuales se pueden clasificar en invasivas y no invasivas. Las terapias farmacológicas, cognitivo conductuales y el uso dispositivos interoclusales se encuentran dentro de la categoría de terapias no invasivas. Los dispositivos interoclusales tipo pívot, han sido ampliamente utilizados en el alivio de artralgias de ATM. Se describe un $70 \%$ de alivio del dolor con el uso de éstos dispositivos en pacientes con artralgias de ATM. El objetivo de esta revisión es evaluar la evidencia encontrada respecto de los dispositivos tipo pívot bilaterales y sus resultados como tratamiento en artralgias de ATM.

Palabras clave: Articulación temporomandibular, artralgia, trastornos temporomandibulares.

\section{SUMMARY}

Arthralgia of Temporomandibular Joint is a term used in the Diagnostic Criteria for Temporomandibular Disorders (DC/TMD), defined as pain of joint origin that is affected by jaw movement, function, or parafunction, and replication of this pain occurs with provocation testing of the TMJ. In the management of Arthralgia the evidence describes different types of therapies, which can be classified as invasive and non-invasive. The pharmacological and cognitive behavioural therapies, as well as the use of interocclusal appliances are considered among the non-invasive therapies. The interocclusal pivot appliances have been widely used to relieve the TMJ arthralgia. The action mechanism of these appliances would be produced by a distraction of the TMJ as a result of the downward movement of the mandibular condylar process; as an added effect it also lowers the

* Cirujano Dentista. Alumno Especialidad y Magíster en Trastornos Temporomandibulares y Dolor Orofacial. Facultad de Odontología. Universidad de La Frontera. Temuco, Chile.

** Cirujano Dentista. Especialista y Magíster en Trastornos Temporomandibulares y Dolor Orofacial. Departamento de Odontología Integral del Adulto. Facultad de Odontología. Universidad de La Frontera. Temuco, Chile.

*** Centro de investigación CIMOFIR Facultad Odontología. Universidad de La Frontera. Temuco, Chile.

**** Cirujano Dentista. Especialista en Trastornos Temporomandibulares y Dolor Orofacial. Magíster en Odontología mención Trastornos Temporomandibulares y Dolor Orofacial. Departamento de Odontología Integral del Adulto. Facultad de Odontología, Universidad de La Frontera. Temuco, Chile. 
intrarticular pressure. Current evidence describes a $70 \%$ of pain relief in patients with TMJ arthralgia. The aim of this review is evaluating the evidence found in bilateral pivot appliances and their results as a treatment for TMJ arthralgia.

Key words: Temporomandibular joint, arthralgia, temporomandibular joint disorders.

Fecha de recepción: 10 de febrero de 2015.

Aceptado para publicación: 5 de marzo de 2015.

Cerda-Trujillo C, Iturriaga V, Fuentes R, Bornhardt T. Manejo de artralgia de articulación temporomandibular mediante la utilización de dispositivos interoclusales tipo pívot; revisión de la literatura. Au. Odontoestomatol 2015; 31 (5): 299-304.

\section{INTRODUCCIÓN}

Artralgia es un término utilizado en los criterios diagnósticos para los trastornos temporomandibulares (CD/TTM), la cual es definida como un dolor de origen articular que se ve aumentado por el movimiento mandibular, ya sea en función o parafunción, y la replicación de este dolor mediante la palpación de la ATM (1). El 10 a 15\% de la población presenta trastornos temporomandibulares (TTMs) con sintomatología dolorosa (2), de los cuales la mayoría de los casos se producen en adultos jóvenes en edades comprendidas entre 20 y 40 años (3). En cuanto a la distribución en la población, el género femenino representa más del $70 \%$ del total de pacientes que consultan, sin considerar la causa de la artralgia (4). Como manejo de la artralgia se describen diferentes niveles de tratamiento los cuales pueden ser aplicados en conjunto y de acuerdo a la patología específica diagnosticada (2).

En relación a las terapias comúnmente descritas se reconocen dos grupos, las de tipo invasivas y las no invasivas. Dentro de las terapias no invasivas podemos agrupar la educación al paciente de su diagnóstico, pronóstico y tratamiento, el manejo de dieta, ejercicios de movilización mandibular, fisioterapia, el uso de dispositivos interoclusales entre otros (5).

Es común, dentro de la evidencia, el uso de dispositivos interoclusales como manejo del dolor que afecta la ATM, describiéndose diferentes tipos según el objetivo que se requiera (6), sin embargo, la presente revisión se enfocará sólo a la evidencia disponible respecto de los dispositivos interoclusales tipo pívot.

\section{METODOLOGÍA}

Se realizó una búsqueda bibliográfica dentro de las bases electrónicas de datos EBSCO Dentistry and Oral Sciences Source, MEDLINE y LILACS. Los términos de búsqueda utilizados fueron "Temporomandibular Joint" [MeSH Terms], Temporomandibular Joint Disorders [MeSH Terms], "Arthralgia" [MeSH Terms] y "Oral Appliance" [All Fields], siendo empleado el término boleanos AND para su unión. Se restringió la búsqueda sólo por idioma, incluyendo artículos en español e inglés. Se seleccionaron artículos por pertinencia según el título, resumen y finalmente texto completo.

\section{DISPOSITIVOS INTEROCLUSALES TIPO PÍVOT}

Previa a la aparición de los dispositivos distractores en el territorio orofacial, se describe el efecto positivo de dispositivos a nivel de articulaciones vertebrales, los cuales también tiene como objetivo lograr una distracción articular en casos agudos de prolapsos discales en columna (7). En 1950, comienza la era de los dispositivos interoclusales tipo pívot, los cuales son descritos por Víctor Sears en 1956 (8). Posteriormente, es evaluado su efecto en 1978 por Lous en una muestra de 60 pacientes con un 71\% de satisfacción (7). Los dispositivos tipo pívot son fabricados sobre láminas de acetato duro de $1 \mathrm{~mm}$ de grosor (0,6 gauge), las cuales son adaptadas al modelo de yeso por medio de una máquina de vacío. Posteriormente con acrílico de autocurado transparente se ubican los pívot lo más posterior en la arcada, con contactos bilaterales uniformes (9) Figura 1. 


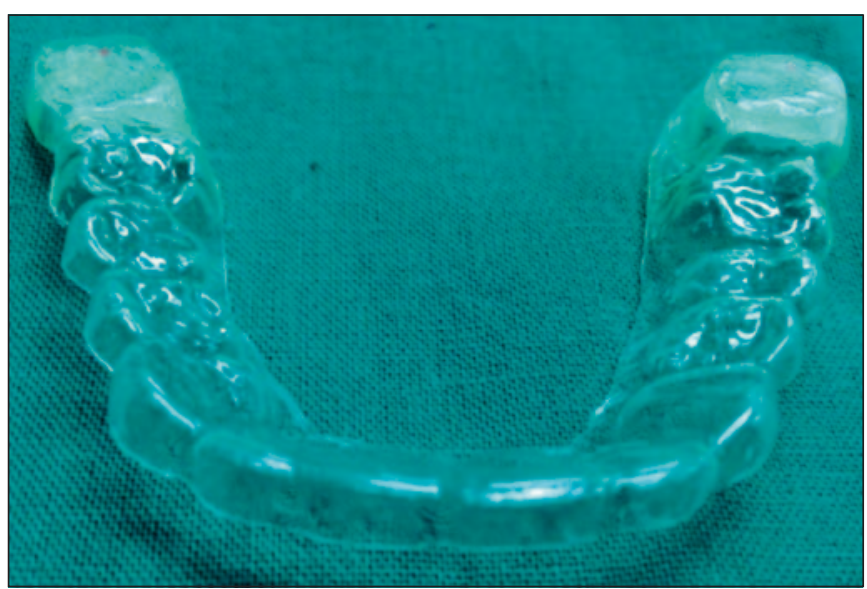

Fig. 1. Dispositivo tipo pívot bilateral; se muestra el esqueleto de acetato y los topes de acrílico de autocurado.

\section{MECANISMO DE ACCIÓN}

Si se evalúa el movimiento de cierre mandibular y se proyecta a un sistema de palancas, en un cierre normal se puede realizar una palanca de orden III con un punto de apoyo en los componentes de la ATM, es decir, corresponde a la unión del proceso condilar al tubérculo y fosa articular con el disco articular interpuesto entre ellos. El objetivo del dispositivo interoclusal tipo pívot, de disminuir el dolor articular, se produciría por la distracción en la ATM. Se ha demostrado que por medio de la aplicación de una fuerza anterior se puede trasladar el eje de giro de la ATM a los topes de acrílico transformando la articulación en una palanca de orden I debido a la fuerza producida anterior a los topes de acrílico (9). Para lograr este efecto los topes de acrílico se deben ubicar lo más posterior del arco dentario posible, idealmente entre los dos vectores de fuerza anterior y posterior, dados por los músculos maseteros, temporales y pterigoideos mediales (10).

Inicialmente se pensaba que reduciría la presión articular con esta ubicación de los pívot de acrílico, al acercarse los dientes anteriores, sin embargo, este efecto puede ocurrir siempre y cuando los pívots se ubiquen detrás de los músculos que producen la fuerza de cierre, es decir maseteros, temporales y pterigoideos mediales (10). El músculo temporal, que al momento de cierre genera un vector de fuerza con una dirección posterior oblicua superior, al introducir los pívot detiene el proceso coronoides y cambia el punto de apoyo de la ATM a los topes de acrílico, anulando así su vector de fuerza, siempre y cuando los pívots de acrílico se encuentren lo más posterior al eje de fuerza (9) .

Seedorf et al. (2007) afirman que las inserciones musculares del masetero, temporal y pterigoideo medial se encuentran, por lo general, detrás del primer molar por lo que, por más posterior que se ubique el pívot, provocará un torque mandibular con una dirección hacia arriba (6). Pese a lo anterior no se ha llegado a consenso respecto al mecanismo de acción específico o su forma de utilización (9).

\section{¿RESULTA POSIBLE LA DISTRACCIÓN ARTICULAR?}

De acuerdo a la evidencia encontrada, el hecho de que los dispositivos interoclusales tipo pívot bilateral logren una distracción articular es controvertida, Moncayo (1994) encontró que sólo un 30\% de los pacientes presenta una distracción del proceso condilar mandibular de forma bilateral, el 35\% mostró que ambos cóndilos se ubican en una posición más protruida, y 35\% de los sujetos mostró una situación combinada (10). Sato et al. (2000) reportan un desplazamiento superior posterior del cóndilo, sin importar si la posición de los pívots fue anterior o posterior al segundo molar (11). Seedorf et al. (2007) describen un efecto de movimiento hacia caudal del proceso condilar a lo largo del tubérculo articular (6). Behr et al. (2007) realizan un estudio comparativo, con un plazo de 13 años, entre la utilización de dispositivos tipo pívot, estabilización y de reposición anterior, con controles al $1 \stackrel{\circ}{\circ}, 5^{\circ}$ y y $13^{\circ}$ - años, dando como resultado la disminución de los síntomas en un $30 \%$ de los pacientes que utilizaron los dispositivos tipo pívot, sin embargo, tanto la limitación de apertura como la presencia de ruidos articulares vuelven a aumentar en los controles a los 5 y 13 años (12). Linsen et al., en el 2012, realizan un estudio donde comparan la utilización de diferentes dispositivos interoclusales; estabilización, reposicionador anterior y pívots uni y bilaterales, pero además incluye la utilización de una mentonera, el que se describe como una banda elástica, que se ubica alrededor de la cara produciendo una fuerza extraoral a nivel del mentón. Lo anterior es realizado con la 
intención de evitar el efecto protrusivo del proceso condilar y aumentar la fuerza anterior. Sus resultados arrojan una significancia estadística a favor del cambio de posición más inferior que anterior del proceso condilar sólo para el grupo que utilizó el dispositivo pívot bilateral y que incluyen la mentonera (13). El uso de mentonera se presenta por primera vez en 1978 por Lous, donde describen su uso en pacientes que no respondían bien a otras terapias (7).

Demling et al. (2009) comparan la posición de proceso condilar en su fosa con la utilización de un dispositivo tipo pívot, de estabilización y sin ellos, pidiendo al paciente que muerda con mínima y máxima fuerza en 5 ocasiones. Sus resultados arrojan que el movimiento del proceso condilar, con el uso de ambos dispositivos, se dirige en dirección hacia abajo y adelante respecto a la fosa mandibular, por lo que no se podría considerar como una distracción articular, debido a que correspondería a la traslación provocada por el aumento de la distancia interoclusal vertical (14). Respecto a la altura de los dispositivos, Linsen et al. (2012) describen un cambio de posición estadísticamente significativo, hacia anterior inferior y lateral del proceso condilar respecto de la fosa con pívot de $2 \mathrm{~mm}$ de altura, en comparación a los dispositivos de $0,6 \mathrm{~mm}$ de altura. No obstante al comparar ambas alturas de los pívot de acrílico con el uso de la mentonera no arrojan diferencias estadísticamente significativas. Por incomodidad al uso de la mentonera, los autores recomiendan la aplicación de fuerza craneal por medio de los pulgares, con los codos apoyados en una superficie firme durante dos horas al día (13).

\section{INDICACIONES Y EFECTOS ADVERSOS}

Se describen diferentes indicaciones terapéuticas respecto a los dispositivos interoclusales tipo pívot. Un estudio describe el uso permanente de los dispositivos durante 24 horas, exceptuando las comidas, por 3 meses (14). Schmitter et al. (2005) disminuyen el uso a 18 horas, pero por un periodo 6 meses (16). Como también se describe la recomendación de utilizar los dispositivos durante 2 horas diarias siempre y cuando se realice al mismo tiempo fuerza extraoral a nivel del mentón (13). Sin embargo, la fa- bricación de estos dispositivos interoclusales está limitado a la presencia de molares y sus respectivos antagonistas para asegurar el soporte de los pívots y su correcto uso (16).

Sólo una publicación describe la inexistencia de efectos secundarios por el uso de dispositivos tipo pívot bilateral, durante un periodo de 3 meses (15).

\section{DISCUSIÓN}

Producto de la información encontrada, se puede analizar los dispositivos tipo pívot, de la siguiente forma:

1) Al analizar la mecánica del dispositivo, se creía que la disminución de los síntomas era debido a la distracción de la ATM. Se han realizado estudios para cuantificar la dirección y magnitud del desplazamiento del cóndilo en la articulación, los cuales no han llegado a consenso ni tampoco han logrado comprobar este método de acción. Demling et al., con el uso de un registro base con ultrasonido, encuentran un desplazamiento anterior e inferior del proceso condilar en la ATM (14), resultados que son compartidos por Seedorf et al. (6) y Moncayo (10). Sin embargo, Linsen et al. (2012) observan un desplazamiento hacia inferior del cóndilo mediante la utilización de una mentonera (13). De acuerdo con la evidencia revisada, el tema sigue siendo controvertido.

2) Respecto a la altura de los pívots, se describen distintas medidas dependiendo de los autores, rangos desde los 0,3-0,6 $\mathrm{mm}$ a 1,9-2,0 $\mathrm{mm}$. Linsen et al. (2012) determinan que $2 \mathrm{~mm}$ de altura lograrían el efecto deseado del dispositivo con el uso de mentonera (13).

3) Por último, una situación no aclarada son las indicaciones de uso de este dispositivo, Stieschscholz et al. (2005) realizan un estudio en cual compara el uso de dispositivos de estabilización y tipo pívot ambos con indicaciones de uso diurno y nocturno exceptuando las comidas durante 3 meses, donde los dos grupos disminuyeron los síntomas en un plazo de 3 meses (15). Schmitter et al. (16) realizan un ensayo clínico donde compara dispositivos en céntrica (estabilización) con dispositivos distractores (tipo pívot) en pacientes 
con luxación discal anterior sin reducción, diagnosticados mediante resonancia nuclear magnética, por un periodo de 6 meses indicando un uso de 18 horas diarias, el autor describe los dispositivos tipo pívot como menos exitosos en comparación con los dispositivos en céntrica. Linsen et al. (2012) agregan a la evidencia la indicación de uso de 2 horas diarias más la aplicación de fuerza extraoral, siendo esta indicación la más adecuada para una reducción de la artralgia (13).

\section{CONCLUSIÓN}

El principal motivo de consulta en el área de los TTMs es por dolor articular (artralgia). Es imperiosa la resolución rápida y efectiva del cuadro debido al potencial riesgo de sensibilización central o periférica que puede afectar la región orofacial desde las aferentes sensitivas trigeminales. Dado que los tratamientos farmacológicos presentan una gama de reacciones adversas medicamentosas, en ocasiones, hace imposible su indicación en algunos pacientes, por lo que el uso de dispositivos tipo pívot es una excelente herramienta terapéutica. Se describe el uso de mentonera en dos publicaciones, ambas presentando mejores resultados.

A pesar que la evidencia, es controvertido referente a el uso de los dispositivos tipo pívot y que aún no se llega a consenso respecto a su mecanismo de acción se encontró evidencia de que entre el $70 \%$ a $90 \%$ de los pacientes con artralgia son tratados con éxito por medio de esta terapia (11). Debido a la falta de resultados concluyentes en los estudios, es recomendable realizar más y mejores estudios con respecto a la indicación, mecanismo de acción e indicaciones del dispositivo tipo pívot.

\section{BIBLIOGRAFÍA}

1. Schiffman E, Ohrbach R, Truelove E, Look J, Anderson G, Goulet J, et al. Diagnostic Criteria for Temporomandibular Disorders (DC/TMD) for Clinical and Research Applications: Recommendations of the International RDC/TMD Consortium Network* and Orofacial Pain Special Interest Group. J Oral Facial Pain Headache 2014;28:6-27.
2. List T, Axelsson S. Management of TMD: evidence from systematic reviews and meta-analyses. J Oral Rehabil 2010;37:430-51.

3. Maydana A, Tesch R, Denardin O, Ursi W, Dworkin S. Possible etiological factors in temporomandibular disorders of articular origin with implications for diagnosis and treatment. Dental Press J Orthod 2010;15(3):78-86.

4. Kino K, Sugisaki M, Haketa, T, Amemori Y, Ishikawa, $\mathrm{T}$ Shibuya, et al. The comparison between pains, difficulties in function, and associating factors of patients in subtypes of temporomandibular disorders. J Oral Rehabil 2005; 32:315-25.

5. Vos L, Huddleston J, Stegenga, B. Lavage Therapy Versus Nonsurgical Therapy for the Treatment of Arthralgia of the Temporomandibular Joint: A Systematic Review of Randomized Controlled Trials. J Orofac Pain 2013;27(2): 171-8.

6. Seedorf H, Scholz A, Kirsch I, Fenske C, Jude H. Pivot appliances - is there a distractive effect on the temporomandibular joint? J Oral Rehabil 2007;34:34-40.

7. Lous I. Treatment of TMJ sindrome. J Prosthet Dent 1978;40(2):179-82.

8. Sears V. Occlusal pivots. J Prosthet Dent 1956;6: 332-8.

9. Moncayo S. Biomechanics of Pivoting Appliances. J Orofac Pain 1994;8:190-6.

10. Okeson J. Tratamiento con férulas oclusales. En: Okeson J. Tratamiento de oclusión y afecciones Temporomandibulares. ELSEVIER España, 2013.

11. Sato H, Ukon S, Ishikawa M, Ohki M, Kitamori, $\mathrm{H}$. Tomographic Evaluation of TMJ Loading Affected by Occlusal Pivots. Int J Prosthodont 2000;13(5):399-404.

12. Behr M, Stebner K, Kolbeck C, Faltermeier A, Driemel O, Handel G. Outcomes of temporo- 
mandibular joint disorder therapy: observations over 13 years. Acta Odontol Scand 2007;65:24953.

13. Linsen S, Stark H, Matthias A. Changes in condylar position using different types of splints with and without a chinstrap: a case-control study. J Craniomandibular Pract 2012;30(1): 25-31.

14. Demling A, Fauska K, Ismail F, Stiech M. A comparison of change in condylar position in Asymptomatic Volunteers utilizing a stabilization and a pivot appliance. J Craniomandibular Pract 2009;21(1):54-61.

15. Stiesch-scholz M, Kempert J, Wolter S, Tschernitschek H, Rossbach A. Comparative prospective study on splint therapy of anterior disc displacement without reduction. J Oral Rehabil 2005;32:474-9.

16. Schmitter M, Zahran M, Phu Duc JM, Henschel V, Rammelsberg P. Conservative Therapy in Patients With Anterior Disc Displacement Without Reduction Using 2 Common Splints: A Randomized Clinical Trial. J Oral Maxillofac Surg 2005;63:1295-303.

\section{CORRESPONDENCIA}

Camila Cerda-Trujillo

Facultad Odontología

Universidad de La Frontera

Manuel Montt, 115

Temuco. Chile.

Correo electrónico: CamilaACerdaT@gmail.com 